\title{
Noninvasive Mechanical Ventilation Guidelines and Standard Protocols for Noninvasive Mechanical Ventilation in Patients with High-Risk Infections
}

\author{
Stefanie Keymel and Stephan Steiner
}

\author{
Keywords \\ NIV • Guidelines $\bullet$ High-risk infection
}

\subsection{Introduction}

Noninvasive ventilation (NIV) is associated with lower rates of endotracheal intubation and decreased mortality in patients with acute respiratory failure. Therefore, NIV should be preferred to invasive ventilation whenever possible [1]. In clinical settings, most of the patients were treated by NIV because of pulmonary edema or exacerbated chronic obstructive lung disease (COPD) [2]. With endemic and highrisk infection, most of the critically ill patients develop acute lung injury (ALI) and/ or acute respiratory distress syndrome (ARDS). Furthermore, NIV, an "aerosolproducing factor" might be regarded as a high-risk procedure for medical staff [3].

We discuss two issues here: guidelines and protocols for NIV and specific recommendations regarding its use during endemic infections, especially in high-risk infections such as severe acute respiratory syndrome (SARS) or influenza (H1N1 virus).

\footnotetext{
S. Keymel, MD

Medical Faculty, Department of Cardiology, Pneumology and Angiology, Heinrich Heine University Düsseldorf, Moorenstrasse 5, 40225 Düsseldorf, Germany

e-mail: stefanie.keymel@med.uni-duesseldorf.de

S. Steiner, MD $(\bowtie)$

Clinic of Cardiology, Pneumology and Intensive Care Medicine, St. Vincenz Hospital, Auf dem Schafsberg, 65549 Limburg/Lahn, Germany

e-mail: s.steiner@st-vincenz.de
} 
Table 40.1 Indications for the use of NIV based on the current guidelines

\begin{tabular}{ll}
\hline Indications for NIV [9] & Mode \\
\hline $\begin{array}{l}\text { Palliative care in patients not considered for intubation } \\
\text { Acute exacerbated COPD with hypercapnic failure }\end{array}$ & NIV \\
\hline $\begin{array}{l}\text { Cardiogenic pulmonary edema } \\
\text { Hypercapnic respiratory failure due to chest wall deformity or neuromuscular } \\
\text { disease }\end{array}$ & CPAP, NIV \\
\hline $\begin{array}{l}\text { Weaning and postextubation failure } \\
\text { RF in immunocompromised patients }\end{array}$ & CPAP, NIV \\
\hline Improvement of ventilation during bronchoscopy & CPAP, NIV \\
\hline
\end{tabular}

NIV is not generally recommended for the use in acute respiratory failure due to acute respiratory distress syndrome or acute lung injury

$R F$ respiratory failure, CPAP continuous positive airway pressure, $N I V$ noninvasive ventilation, $C O P D$ chronic obstructive pulmonary disease

\subsection{Guidelines and Protocol for NIV in the Acute Care Setting}

As a result of the growing importance of NIV in emergency and intensive care medicine, several guidelines on this topic were published during the last decade. The following overview summarizes the recommendations on NIV in patients with ALI and ARDS, which are known to complicate high-risk infections.

In 2001, an international expert group concluded that NIV may substitute for invasive ventilatory support in patients with hypoxemic respiratory failure due to pneumonia. The authors noted that there were only three randomized studies comparing NIV with invasive ventilation and that they had different endpoints and results [2]. A year later, the British Thoracic Society (BTS) published guidelines on the use of NIV in patients with acute respiratory failure (ARF). They did not consider the treatment of ALI due to respiratory infection. Conversely, at this time severe hypoxemia was regarded as a contraindication for NIV [4]. Certainly, there was no link to high-risk infection at that time. The Canadian Critical Care Trials group made no recommendations about the use of NIV in ARDS patients or those with severe community-acquired pneumonia (CAP) in 2011 [5]. In summary, compared to NIV for exacerbated COPD (hypercapnic respiratory failure), cardiogenic lung edema, or postextubation failure, the data regarding the use of NIV in patients with hypoxemic ARF are less clear [1].

\subsubsection{Indication for NIV}

Tables 40.1 and 40.2 summarize common accepted indications and contraindications for NIV. NIV might be considered in patients with tachypnea and a respiratory rate $>24$ breaths/min, a poor alveolar gas exchange level as indicated by $\mathrm{PaO}_{2} /$ $\mathrm{FiO}_{2}<200 \mathrm{mmHg}$, and/or severe dyspnea accompanied by the use of accessory respiratory muscles [6]. Beyond this, NIV may be undertaken as a therapeutic trial with a view to tracheal intubation if it fails or as a ceiling of treatment in patients who are not candidates for intubation [4]. It should be emphasized that intubation 
Table 40.2 Contraindications for the use of NIV [2, 9]

\begin{tabular}{l}
\hline Cardiac or respiratory arrest \\
\hline Severe encephalopathy \\
\hline Severe upper gastrointestinal bleeding \\
Facial surgery/trauma \\
\hline Inability to cooperate/protect the airway \\
\hline High risk for aspiration \\
\hline
\end{tabular}

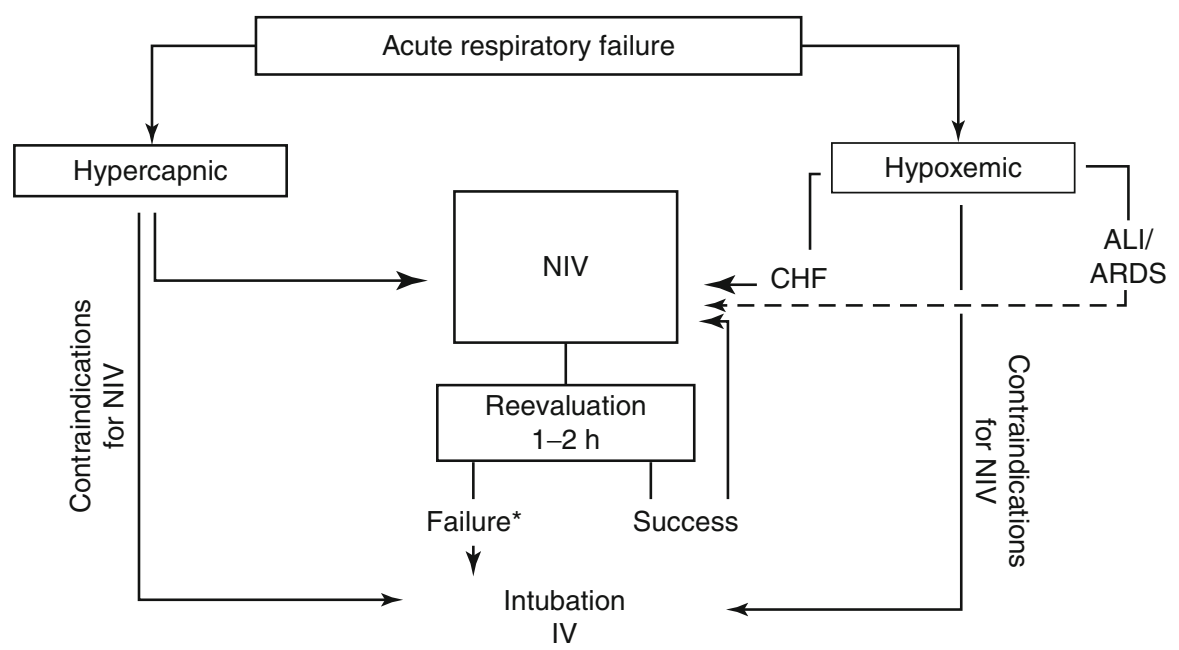

Fig. 40.1 Noninvasive ventilation (NIV) in patients with acute respiratory failure. There is a strong recommendation for NIV in those with hypercapnic respiratory failure and hypoxemic failure associated to cardiogenic edema. None of the guidelines favor NIV in patients with acute lung injury or acute respiratory distress syndrome because of lack of evidence. If NIV is used in these patients, early detection of failed NIV requires careful monitoring. *Signs of NIV failure: worsening of gas exchange, hemodynamic instability, change of mental status, signs of respiratory fatigue. See Table 40.2 for contraindications

should be performed early in patients with pneumonia and ARDS who do worsen or have not improved after 1-2 h [7, 8] (Fig. 40.1).

\subsubsection{Protocol and Practical Approach to NIV}

There is a broad agreement that NIV should be conducted in the intensive care unit (ICU), where immediate expertise is available to enable a rapid transition to invasive ventilation if needed $[1,8,9]$.

\subsubsection{Choice of Interface}

Noninvasive ventilation is defined as ventilator assistance to the lungs without an artificial airway. There are various devices, including negative-pressure ventilators 
(e.g., the so-called tank ventilator, or "iron lung"), several masks, and helmets. Because of limited practicability, tank ventilators do not play a major role in modern intensive care medicine. Selection of the optimal interface-which connects the ventilator to the nose, mouth, or both-is an essential part of NIV. Air leakage, discomfort, or claustrophobia might result in patient intolerance. In the acute care setting, nasal, oronasal, or full-face masks are primarily used $[1,4,5]$. There are few randomized controlled trials comparing the use of an oronasal mask with a nasal mask. Nevertheless, the oronasal mask has been better tolerated than nasal mask or full-face mask $[1,10]$. Because there is a lack of evidence regarding which interface is best, some guidelines do not give recommendations about the use of interfaces [5]. Others favor the use of a full-face mask for the first $24 \mathrm{~h}$, switching to a nose mask if preferred by the patient $[4,11]$.

In general, masks and exhalation valves that are licensed as reusable by the manufacturer require high-level disinfection. They should be disassembled in their parts and then undergo an automatic process using washer, disinfector, and dryer.

Attaching a bacterial filter to the ventilator's output can minimize respirator contamination [4]. As an alternative, using single-use material could reduce the risk of infection.

\subsubsection{Mode of NIV}

Noninvasive ventilation can be performed using pressure support ventilation, proportional assist ventilation, or volume-controlled ventilation [1, 2]. Schönhofer et al. [1] recommended the use of positive-pressure ventilation with inspiratory pressure support and positive end-expiratory pressure (PEEP). As patients with ARF are often agitated and have pronounced respiratory drive, ventilation triggered by the patient's own respiratory efforts is beneficial compared to controlled, timebased ventilation. When there is not sufficient spontaneous inspiratory effort or it is inadequate to trigger the ventilator, pressure-controlled ventilation could be used $[1,2]$. Other guidelines do not emphasize a mode of ventilation on the strength of insufficient evidence [5]. Similar to invasive ventilation, ventilator settings should be adjusted to provide the lowest inspiratory pressures or volumes needed to improve oxygenation and patient comfort, which can be estimated by the decrease in the respiratory rate and respiratory muscle unloading [2]. Because most of the critical ill patients with SARS or H1N1 virus infection develop ARDS and ALI, a lung-protective ventilatory strategy and fluid restriction are essential.

\subsubsection{Clinical Course and NIV Failure}

The most important parameters during the clinical course are $\mathrm{PaCO}_{2}$ (arterial partial pressure of carbon dioxide), $\mathrm{pH}$, respiratory rate, dyspnea, and alertness. The aforementioned parameters have to show a trend toward improvement during the first $2 \mathrm{~h}$ of NIV [1]. The NIV failure rate in patients with hypoxic respiratory failure is 
estimated to be $30 \%$ (CAP) to $50 \%$ (ARDS) [7, 9, 12]. Failure occurs early or after a few days [1]. It should be noted that NIV failure is associated with a worse outcome, which might be a consequence of a delayed response to the NIV failure because of inadequate monitoring or delayed definitive care [13]. Other predictors of failure are the duration of NIV, oxygenation index, and the Simplified Acute Physiology Score II at admission, and, as expected, the length of ICU stay [7]. Other authors found a high APACHE score, copious respiratory secretions, poor nutritional status, and confusion or impaired consciousness to be associated with NIV failure [2].

\subsection{Specific Recommendations for Using NIV in Patients with Endemic and High-Risk Infections}

There are specific problems concerning the use of NIV in patients with endemic and high-risk infections. First, there are no controlled trials on this topic. Therefore, recommendations are largely based on supposition [3]. It is of concern that NIV, as an "aerosol-producing procedure," possibly increases the risk of caregiver exposure or of exposure to other patients, which would be disastrous in case of a pandemic. Therefore, organizations such as the World Health Organization [14] and the UK National Health Services Agency [15] published guidelines that treat NIV as a high-risk procedure. Nevertheless, there are no controlled data comparing particle dispersion between individuals undergoing NIV and those who are not. Furthermore, it should be kept in mind that endotracheal intubation also is at risk of transmitting disease.

In an experimental model, Hui and coworkers [16] found that flow from a noninvasive ventilator may increase occupational risk. As this risk may be mediated by air leaks, fitting the mask properly is essential. Full-face masks and helmets might be superior to nasal masks.

Also, NIV must be managed under strict isolation measures with adequate protection (e.g., N-95 mask) of the health care workers who attend to the patients. As far as possible, infected patients should be isolated in rooms with negative pressure.

Although most of the guidelines do not recommended use of NIV, it has become part of the standard treatment protocol for SARS [17]. Han et al. [18] demonstrated that NIV was not only effective in avoiding intubation and invasive ventilation, it effectively reduced the ICU length of stay. No infection was detected in 155 health care workers, and their serology tests for coronavirus were negative.

\section{Key Major Recommendations}

- Current guidelines do not recommend NIV for the treatment of hypoxemic respiratory failure in endemic and pandemic infections (e.g., SARS or H1N1). However, the level of evidence is low.

- Noninvasive ventilation appears to be a reasonable option in carefully selected cases, which should be treated under optimal conditions with awareness of NIV failure and might be regarded as a high risk procedure for medical staff. 


\section{References}

1. Schönhofer B, Kuhlen H, Neumann P, Westhoff M, Berndt C, Sitter H. Clinical practice guidelines: non invasive ventilation as treatment of acute ventilation. Dtsch Arztebl Int. 2008;105(24):424-33.

2. Evans T, Albert R, Agnus D, et al. International consensus conference in intensive care medicine: noninvasive positive pressure ventilation in acute respiratory failure. Am J Respir Crit Care Med. 2001;163:283-91.

3. McCracken J. Should noninvasive ventilation be considered a high-risk procedure during an epidemic? CMAJ. 2009;181(10):663-4.

4. British Thoracic Society Standards of Care Committee. Non-invasive ventilation in acute respiratory failure. Thorax. 2002;57(192):211.

5. Keenan S, Sinuff T, Burns K, et al. Clinical practice guidelines for the use of nonivasive positive-pressure ventilation and nonivasive continuous positive airway pressure in the acute care setting. CMAJ. 2011;183(3):195-213.

6. Carbonara P, Nava S. Noninvasive mechanical ventilation. In: Spiro SG, Silvestri GA, Agusti A, editors. Clinical respiratory medicine. Elsevier; 2012. p. 431-6.

7. Carron M, Freo U, Zorzi M, Ori C. Predictors of failure of noninvasive ventilation in patients with severe community-acquired pneumonia. J Crit Care. 2010;25(540):e9-14.

8. Schönhofer B, Kuhlen R, Neumann P, Westhoff M, Berndt C, Sitter H. Non-invasive mechanical ventilation in acute respiratory failure. Pneumologie. 2008;62:449-79.

9. Lim W, Baudouin S, George R, et al. BTS Guidelines for the management of community acquired pneumonia in adults: update 2009. Thorax. 2012;64(Suppl III):1-55.

10. Girault C, Briel A, Benichou J. Interface strategy during non invasive pressure ventilation for hypercapnic acute respiratory failure. Crit Care Med. 2009;37:124-31.

11. Roberts C, Brown J, Reinhardt A, et al. Non-invasive ventilation in chronic obstructive pulmonary disease: management of acute type 2 respiratory failure. Clin Med. 2008;8(517):521.

12. Antonelli M, Conti G, Moro M, et al. Predictors of failure of noninvasive positive pressure ventilation in patients with acute hypoxemic respiratory failure: a multi-center study. Intensive Care Med. 2001;27:1718-28.

13. Bersten A. Best practices for noninvasive ventilation. CMAJ. 2011;183(3):293-4.

14. WHO Interim Guidelines. Infection prevention and control of epidemic- and pandemic-prone acute respiratory diseases in health care. 2007. www.who.int/csr/resources/publications/WHO_ CDS_EPR_2007_6c.pdf.

15. Pandemic H1N1 2009 influenza: clinical management guidelines for adults and children. Guidance Department of Health, UK Guidelines. 30 Oct 2009. p. 12904.

16. Hui D, Hall S, Mathew T, et al. Noninvasive positive pressure ventilation an experimental model to assess air and particle dispersion. Chest. 2006;130:730-40.

17. Cheung T, Yam L, So L, et al. Effectiveness of noninvasive positive pressure ventilation in the treatment of acute respiratory failure in severe acute respiratory syndrome. Chest. 2004;126:845-50.

18. Han F, Jiang Y, Zheng J, Gao Z, He Q. Noninvasive positive pressure ventilation treatment for acute respiratory failure in SARS. Sleep Breath. 2004;8(2):97-106. 